\title{
PERFIL CLÍNICO-EPIDEMIOLÓGICO DOS PACIENTES DA UNIDADE DE TERAPIA INTENSIVA PEDIÁTRICA DE UM HOSPITAL REFERÊNCIA EM TRAUMA NA AMAZÔNIA
}

Gabriela Martins de LIMA ${ }^{1}$

Fabiano José da Silva BOULHOSA ${ }^{2}$

Juliana Anezia Rodrigues de SOUZA ${ }^{3}$

Kéven Lorena de Paula GONÇALVES ${ }^{4}$

Leonardo Ramos Nicolau da COSTA ${ }^{5}$

Manuelle Vieira NICOLAU ${ }^{6}$

Paola Katherine Esteves da SILVA ${ }^{7}$

Patrícia Gazel PICANÇO ${ }^{8}$

Rafaela Cordeiro de MACÊDO ${ }^{9}$

Rodrigo Alcântara Carnevalli de ARAÚJO ${ }^{10}$

\begin{abstract}
${ }^{1}$ Mestranda, supervisora do serviço de Reabilitação do Hospital Metropolitano de Urgência e Emergência.gabimlima@gmail.com

${ }^{2}$ Mestrando, preceptor do Programa de Residência Multiprofissional em Saúde em Urgência e Emergência no Trauma Universidade do Estado do Pará. fabiano.boulhosa@gmail.com

${ }^{3}$ Especialistaem Saúde em Urgência e Emergência no Trauma - Universidade do Estado do Pará. julianadoamaral@ymail.com

${ }^{4}$ Especialista, preceptora do Programa de Residência Multiprofissional em Saúde em Urgência e Emergência no Trauma - Universidade do Estado do Pará. kevenlorenadepaula@gmail.com

${ }^{5}$ Mestre, coordenador do Programa de Residência Multiprofissional em Saúde em Urgência e Emergência no Trauma Universidade do Estado do Pará. leorcosta@gmail.com

${ }^{6}$ Especialista em Saúde da Mulher e da Criança - Universidade do Estado do Pará. manuellenicolau@ hotmail.com

${ }^{7}$ Fisioterapeuta Residente do Programa de Residência Multiprofissional em Saúde em Urgência e Emergência no Trauma- Universidade do Estado do Pará.paolaestevessilva@yahoo.com.br

${ }^{8}$ Fisioterapeuta Residente do Programa de Residência Multiprofissional em Saúde em Urgência e Emergência no Trauma- Universidade do Estado do Pará. patrícia_gazel@hotmail.com

${ }^{9}$ Mestrando, preceptor do Programa de Residência Multiprofissional em Saúde em Urgência e Emergência no Trauma Universidade do Estado do Pará. rafaela_cmacedo@hotmail.com

${ }^{10}$ Especialista- preceptores do Programa de Residência Multiprofissional em Saúde em Urgência e Emergência no Trauma - Universidade do Estado do Pará. rodrigo_carnevalli@yahoo.com.br
\end{abstract}

Recebido em: 22/09/2015 - Aprovado em: 18/07/2016 - Disponibilizado em: 18/12/2016

RESUMO: A terapia intensiva pediátrica (UTI) tem o intuito de prover o cuidado ideal às crianças em grave estado de saúde. Objetivou-se verificar o perfil clínico-epidemiológico dos pacientes admitidos na UTI Pediátrica e Adolescente do Hospital Metropolitano de Urgência e Emergência no ano de 2010. Estudo transversal, retrospectivo, descritivo, com coleta em 132 prontuários, que foram analisados no período de janeiro a março de 2011, no setor de arquivamento de prontuários do HMUE, por meio de ficha de coleta de dados. Dos 132 prontuários coletados, observou-se que o sexo mais prevalente dentre os pacientes internados em 2010, foi o sexo masculino, tendo o trauma maior ocorrência na idade de 1 a 3 anos (30,3\%). O principal diagnóstico de admissão foi o trauma cranioencefálico (36,4\%), seguido de politrauma $(24,2 \%)$ e queimaduras $(15,9 \%)$. No que diz respeito aos municípios de maior procedência de pacientes, o município de Belém destacou-se com 27 casos (20,5\%). A média de permanência dos pacientes no serviço foi de 20 dias, e a maior taxa de mortalidade ocorreu após 24 horas de internação. Quanto aos pacientes que fizeram uso de ventilação mecânica invasiva, 73 pacientes foram ventilados artificialmente, principalmente em decorrência do rebaixamento do nível de consciência.Dessa forma, este estudo vem demonstrar o perfil clínico-epidemiológico de pacientes admitidos em um hospital referência em trauma, favorecendo o entendimento desta problemática, podendo 
embasar estudos locais, e, sobretudo, fomentar estratégias de solução para a minimização de tais injúrias, muitas delas incapacitantes.

DESCRITORES: Perfil clínico-epidemiológico. Traumatismo na infância. UTI pediátrica. Respiração artificial. Traumatismo.

ABSTRACT: The pediatric intensive care unit (ICU) aims to provide optimal care to children in serious health condition. The objective was to verify the clinical and epidemiological profile of patients admitted to the Pediatric ICU and Emergency Department of Hospital Metropolitano teenager in 2010. Cross-sectional study, retrospective, descriptive, collected in 132 records, which were analyzed in the period from January to March 2011, the archiving sector HMUE the records through data collection form. The 132 records collected, it was observed that the most prevalent sex among patients hospitalized in 2010, was the male with the most trauma occurring at age 1-3 years $(30.3 \%)$. The main intake diagnosis was traumatic brain injury (36.4\%), followed by polytrauma $(24.2 \%)$ and burns (15.9\%). With regard to the municipalities of origin of most patients, the municipality of Bethlehem stood out with 27 cases $(20.5 \%)$. The average stay of patients in service was 20 days, and the highest mortality rate occurred after 24 hours of admission. As for the patients who used invasive mechanical ventilation, 73 patients were artificially ventilated, mainly due to the level of consciousness relegation. Thus, this study demonstrates the clinical and epidemiological profile of patients admitted to a hospital reference in trauma, favoring the understanding of this problem and can to base local studies, and, above all, promote solving strategies to minimize such injuries, many of them disabling.

KEYWORDS: Epidemic clinic profile. Childhood traumatism. Pediatric Intensive Care.Respiration, artificialTraumatism.

\section{Introdução}

A terapia intensiva pediátrica no Brasil constitui uma especialidade relativamente jovem. As primeiras unidades de terapia intensiva (UTI) para o público infantil foram inauguradas na década de 70, com o intuito de prover o cuidado ideal às crianças em grave estado de saúde, de tal forma a propiciar a cura das doenças, bem como favorecer $o$ crescimento em direção a uma vida útil, com o pleno desenvolvimento de suas potencialidades, de acordo com Molina et al (2008).

A procura de assistência à pacientes pediátricos dá-se principalmente pelo agravo de doenças respiratórias, estados convulsivos, intoxicações, processos traumáticos e infecciosos, sendo os dois últimos os maiores causadores das admissões nessas unidades (EINLOFT et al, 2002; MOLINA et al, 2008).
De acordo com Rocha (2007), os processos traumáticos são considerados como uma das principais causas de morte e de sequelas em crianças e adolescentes em todo o mundo. Nopaís, as entidades traumáticas são a principal causa de óbito em crianças acima de cinco anos de idade e representam mais de $50 \%$ dos óbitos na adolescência.

$$
\text { Abramovici e Waksman (2005), }
$$
afirmam que dentre os principais traumas que levam a graves complicações clínicas pode-se citar as quedas, as queimaduras, aspiração de corpo estranho, afogamentos, atropelamentos e na fase da adolescência, a violência, seja ela homicídio ou suicídio, que muitas vezes assume importante papel sobrepondo-se as demais causas.

Os principais acidentes estão relacionados ao sexo masculino, pois esse público está mais propenso a se envolver em 
situações que levam ao risco de agravos. Outros fatores de risco como a localização geográfica, onde em cada região predomina certo tipo de acidente; os fatores sócioeconômicos, relacionados com a baixa renda familiar; a escolaridade deficiente dos pais e os ambientes hostis, também podem ser classificados como fatores de risco evidentes para a ocorrência de acontecimentos traumáticos (TASKER; GUPTA; WHITE, 2004 apud ROCHA, 2007).

Os motivos que levam as crianças a estarem mais suscetíveis a estes fenômenos traumáticos são as inabilidades perceptivas, cognitivas e motoras inerentes as diferentes fases de desenvolvimento que as mesmas apresentam. Vale ressaltar que tais riscos estão relacionados com as diferentes faixas etárias, onde em cada uma predomina um tipo de trauma (SCHVARTSMAN; CARRERA; ABRAMOVICI, 2005 apud ROCHA, 2007).

Segundo Filho, Reschke e Horner (2006), outro grande motivo de admissão nas Unidades de Terapia Intensiva Pediátrica (UTI-PED) dá-se por processos infecciosos que podem ocorrer pela agressão de patógenos ainda na comunidade ou dentro do próprio ambiente hospitalar, sendo está última denominada como infecção nosocomial e classificada como a de maior ocorrência nos pacientes internados em Unidade de Terapia Intensiva (UTI) devido à severidade da doença de base como, por exemplo, a severidade do trauma.
Molina et al (2008), cita que é necessário uma equipe multidisciplinar, qualificada para atuar em toda e qualquer situação dentro de uma UTI Pediátrica. Dentro desse novo paradigma de saúde, a fisioterapia vem se tornando essencial nas unidades de terapia intensiva, onde o fisioterapeuta apresenta hoje uma missão primordial de cooperação na Terapia Intensiva. Por meio da aplicação de meios terapêuticos físicos, que objetivam diminuir as complicações e o período de hospitalização, este profissional desempenha importante papel na prevenção, eliminação, manutenção ou melhora de estados psicopatológicos do homem em qualquer fase do seu ciclo de vida (NICOLAU; LAHÓZ, 2007).

A partir desses dados da literatura científica, procurou-se traçar um perfil clínico-epidemiológico dos pacientes admitidos na Unidade de Terapia Intensiva Pediátrica e Adolescente do Hospital Metropolitano de Urgência e emergência no ano de 2010.

\section{Metódos}

Esta pesquisa seguiu os preceitos da Declaração de Helsinque e do Código de Nuremberg, respeitando as Normas de Pesquisa Envolvendo Seres Humanos (Res. CNS 196/96) do Conselho Nacional de Saúde, sendo submetido e aprovado pelo Comitê de Ética do Centro Universitário do Pará sob registro CAAE: 5306.0.000.323-10. 
Tratou-se de um estudo transversal, do tipo retrospectivo, descritivo, com dados coletados em prontuários, de todos os pacientes admitidos na UTI Pediátrica e Adolescente do Hospital Metropolitano de Urgência e Emergência (HMUE) no ano de 2010.

Pacientes com mais de uma admissão no setor, foram incluídos na pesquisa, considerando cada uma das internações como independentes.

A amostra constou de 132 prontuários, realizada no setor de arquivamento de prontuários do Hospital Metropolitano de Urgência e Emergência, localizado na Rodovia BR-316, km 3, no município de Ananindeua (PA), no período de Janeiro a Março de 2011, em horário vespertino.

A coleta dos dados foi realizada por meio de uma ficha elaborada pelos autores da pesquisa, na qual continha tópicos relacionados aos aspectos demográficos (idade, sexo e procedência), diagnóstico de admissão, mecanismo do trauma, tempo de internação, analisada pela média da data de admissão e alta da UTIPED; evolução clínica dos pacientes levando em consideração a taxa de mortalidade (menor ou maior que 24 horas) e o perfil dos pacientes que fizeram uso de ventilação mecânica invasiva, preenchidos com base nas informações contidas nos prontuário de cada paciente da UTI Pediátrica e Adolescente do HMUE em 2010 .

A idade foi codificada em categorias de acordo com a Organização Mundial de Saúde; menores de um ano, de um a três anos, de quatro a seis anos, de sete a nove anos e de dez a quatorze anos (OMS, 2003).

No caso específico deste estudo, não foi possível a obtenção do Termo de Consentimento Livre e Esclarecido dos pacientes pediátricos, visto que se tratou de um estudo com dados retrospectivos do ano de 2010, coletados em prontuários já arquivados.

Após a realização da coleta, todos os dados foram digitados e tabulados em um banco de dados para a execução da análise estatística dos mesmos. O banco de dados, bem como as tabelas e gráficos foram construídos no MicrosoftExcel® 2007. Para análise da significância estatística dos resultados obtidos utilizou-se o teste Quiquadrado, sendo considerado o nível $\alpha=0,05$ (95\%), sendo tais análises executadas por meio do softwareBioEstat ${ }^{\circledR} \quad 5.0$. 


\section{Resultados e Discussão}

Dentre os prontuários alocados na pesquisa, o sexo masculino mostrou-se prevalente entre os casos de admissão na
UTIPed do HMUE, compreendendo 91 dos 132 prontuários pesquisados, o que corresponde a $68,9 \%$, como observado na Tabela 1.

Tabela 1 - Sexo dos pacientes admitidos na UTIPED do HMUE no ano de $2010(n=132)$.

\begin{tabular}{ccc}
\hline \multirow{2}{*}{ Sexo } & \multicolumn{2}{c}{ Freqüência } \\
\cline { 2 - 3 } & $\mathbf{N}$ & $\mathbf{\%}$ \\
\hline Masculino & 91 & 68,9 \\
Feminino & 41 & 31,1 \\
\hline Total & $\mathbf{1 3 2}$ & $\mathbf{1 0 0 , 0}$ \\
\hline
\end{tabular}

Fonte: Prontuários da UTIPED do HMUE, 2010.

Segundo o estudo de Bemet al, (2008), das 387 crianças internadas, pôde-se verificar uma maior prevalência do sexo masculino, representando cerca de $66,4 \%$, caso também observado no estudo de Rocha (2007), onde dos 372 prontuários, foi predominante as crianças do sexo masculino fatos estes que coincidem com o achado do presente estudo, quanto ao sexo dos indivíduos.

Segundo Bem et al (2008) e Rocha (2007), a prevalência de traumas em crianças e adolescentes do sexo masculino, decorre de uma questão sócio-cultural, em que os homens tem a obrigação de proteger a família, bem como o gosto aguçado por situações diferentes que na maioria das vezes os expõe ao perigo.

No que diz respeito à faixa etária dos pacientes admitidos no serviço no ano de 2010, verificou-se que a prevalência entre os casos esteve de 1 a 3 anos de idade, compreendendo $30,3 \%$ da amostra estudada. (Tabela 2)

Tabela 2 - Faixa etária dos pacientes admitidos na UTIPED do HMUE no ano de 2010 (n=132).

\begin{tabular}{cccc}
\hline Faixa etária & $\mathbf{N}$ & Freqüência \\
\cline { 3 - 4 } & & 14 & 10,6 \\
\hline & 1 a 3 anos & 40 & 30,3 \\
4 a 6 anos & 20 & 15,2 \\
\hline
\end{tabular}




\begin{tabular}{ccc}
\hline 7 a 9 anos & 25 & 18,9 \\
10 a 14 anos & 33 & 25,0 \\
\hline Total & $\mathbf{1 3 2}$ & $\mathbf{1 0 0 , 0}$ \\
\hline Fonte: Prontuários da UTIPED do HMUE 2010.
\end{tabular}

Fonte: Prontuários da UTIPED do HMUE, 2010.

De acordo com estudo feito por Rocha (2007), dos 372 prontuários, 148 eram de crianças na faixa etária de 5 a 9 anos; está em desacordo com estudo realizado por Bem et al (2008), onde 151 das 387 crianças admitidas em um hospital estudado por ele possuíam faixa-etária de 2 a 5 anos.

Bem et al (2008), afirmam esses dados descrevendo que quanto mais jovem e imatura a criança, menor sua percepção de risco e maior sua vulnerabilidade e dependência de terceiros, predominando assim os agravos em menores de 5 anos.

O principal diagnóstico de admissão das crianças admitidas na UTIPED do HMUE em 2010 foi o trauma cranioencefálico (TCE), presente em 48 prontuários $(36,4 \%)$. Em menor escala, destacaram-se o politrauma em 32 prontuários $(24,2 \%)$, as queimaduras com 21 ocorrências $(15,9 \%)$, seguidos do trauma abdominal (4,5\%), insuficiências respiratórias $(4,5 \%)$, e trauma torácico $(2,3 \%)$. (Tabela 3$)$

Tabela 3 - Diagnóstico dos pacientes admitidos na UTIPED do HMUE no ano de 2010 (n=132).

\begin{tabular}{|c|c|c|}
\hline Diagnóstico & Quantidade & \% Quantidade \\
\hline TCE & 48 & $36,4 \%$ \\
\hline Politrauma & 32 & $24,2 \%$ \\
\hline Queimadura & 21 & $15,9 \%$ \\
\hline Trauma Abdominal & 6 & $4,5 \%$ \\
\hline Insuficiência Respiratória (IRpA) & 6 & $4,5 \%$ \\
\hline Trauma Torácico & 3 & $2,3 \%$ \\
\hline Asfixia Mecânica+Hemorragia Pulmonar & 1 & $0,8 \%$ \\
\hline Atresia de Mandíbula & 1 & $0,8 \%$ \\
\hline Choque Hipovolêmico & 2 & $1,5 \%$ \\
\hline Epilepsia Pós -Trauma & 1 & $0,8 \%$ \\
\hline Fratura de Antebraço & 1 & $0,8 \%$ \\
\hline Fratura de Fêmur & 1 & $0,8 \%$ \\
\hline Fratura Exposta de Bacia & 1 & $0,8 \%$ \\
\hline
\end{tabular}




\begin{tabular}{lcc} 
Fratura Exposta de Punho+IRpA & 1 & $0,8 \%$ \\
\hline Intoxicação Exógena+Bronquite & 1 & $0,8 \%$ \\
Má Formação Congênita (Espinha Bífida) & 1 & $0,8 \%$ \\
Mal Convulsivo & 1 & $0,8 \%$ \\
Trauma de Tecidos Moles & 1 & $0,8 \%$ \\
Trauma Retal & 1 & $0,8 \%$ \\
TRM Cervical & 1 & $0,8 \%$ \\
\hline Tumoração+Trauma de Língua & 1 & $\mathbf{1 0 0 , 0}$ \\
\hline Total Geral & $\mathbf{1 3 2}$ & \\
\hline
\end{tabular}

Fonte: Prontuários da UTIPED do HMUE, 2010- SAME.

Lacerda (2010), em seu estudo afirma que o TCE é a principal injúria que acomete crianças vítimas de traumas, em cerca de $48,45 \%$ dos casos, comprovação esta que se assemelha ao encontrado por Rocha (2007), o qual afirma que $60 \%$ das causas de admissões na UTIP foram por tal diagnóstico.

Freitas et al (2007), explica tais dados baseando-se nas características peculiares das crianças, como falta de discernimento necessário para lidar com diversas situações e falta de experiência dos responsáveis, que os fazem pensar que as crianças estão aptas para desenvolver determinada ação e na verdade elas não estão preparadas o suficiente, resultando assim no acontecimento de tais fatalidades.

Em estudo de Lacerda (2010), 76,85\% das crianças que foram diagnosticadas com TCE, foram vítimas de quedas, sendo os acidentes automobilísticos a segunda maior ocorrência do TCE, corroborando com o presente estudo onde tal trauma resultou principalmente de quedas $(62,5 \%)$, e em menor frequência de acidentes de trânsito $(25,0 \%)$ (Tabela 4).

Tabela 4 - Diagnóstico x Tipos de Acidentes dos pacientes admitidos na UTIPED do HMUE no ano de 2010 (n=132).

\begin{tabular}{cccccccc}
\hline & \multicolumn{7}{c}{ Diagnósticos } \\
\cline { 2 - 6 } Tipos de Acidentes & \multicolumn{2}{c}{ TCE Grave } & Politrauma & Grande Queimado & Total \\
\cline { 2 - 6 } & $\mathbf{N}$ & $\mathbf{\%}$ & $\mathbf{N}$ & $\mathbf{\%}$ & $\mathbf{N}$ & $\mathbf{\%}$ \\
\hline Queda & 30 & 62,5 & 5 & 15,6 & 0 & 0,0 & 35 \\
Acidente de Trânsito & 12 & 25,0 & 24 & 75,0 & 0 & 0,0 & 36 \\
FAF & 1 & 2,1 & 2 & 6,3 & 0 & 0,0 & 3 \\
Líquido Quente & 0 & 0,0 & 0 & 0,0 & 3 & 14,3 & 3 \\
\hline
\end{tabular}




\begin{tabular}{cccccccc}
\hline Liquido Inflamável & 0 & 0,0 & 0 & 0,0 & 12 & 57,1 & 12 \\
Brasa & 0 & 0,0 & 0 & 0,0 & 1 & 4,8 & 1 \\
Chama & 0 & 0,0 & 0 & 0,0 & 3 & 14,3 & 3 \\
Fogos de artifício & 0 & 0,0 & 0 & 0,0 & 1 & 4,8 & 1 \\
Eletricidade & 0 & 0,0 & 0 & 0,0 & 1 & 4,8 & 1 \\
Outras ocorrências & 5 & 10,4 & 1 & 3,1 & 0 & 0,0 & 6 \\
\hline Total & $\mathbf{4 8}$ & $\mathbf{1 0 0 , 0}$ & $\mathbf{3 2}$ & $\mathbf{1 0 0 , 0}$ & $\mathbf{2 1}$ & $\mathbf{1 0 0 , 0}$ & $\mathbf{1 0 1}$ \\
\hline
\end{tabular}

Fonte: Prontuários da UTIPED do HMUE, 2010.

Em relação aos dados expostos acima, Pereira (2010), explica que as quedas estão interligadas com a fase da infância, onde agregado a este fato inclui-se a deficiência de capacidade protetora da família e o desconhecimento dos inúmeros fatores de risco que permeiam o cotidiano da criança.

Quanto às crianças vitimizadas em acidentes de trânsito que foram admitidas na UTIPED do HMUE, o politrauma foi a principal consequência (75\%), estando presente em 24 casos, de uma amostra de 132 prontuários.

Não foi encontrado nenhum dado na literatura que pudesse ser comparado com o presente estudo, porém Lara (2005) em seu estudo mostra que o politrauma foi causado principalmente por acidentes de trânsito, que segundo o autor ocorrem devido o fato de o Brasil ser um país recorde neste tipo de fatalidade tendo a fascinação do homem pela alta velocidade, a falta de infra estrutura em nossas ruas e estradas e a imprudência de nossos motoristas.
No que diz respeito ao tempo de internação na UTIPED do HMUE, obteve-se como média 20 dias de permanência; o que está em contradição com o estudo realizado por Lara (2005), onde o tempo de internação predominou entre 25 horas a 7 dias, devido ao alto índice de mortalidade no estudo, haja vista que grande parcela dos pacientes chegavam a Unidade de Terapia Intensiva em estado crítico, sobrevivendo poucas horas e/ou dias.

Para Carvalho (2007), os pacientes diagnosticados com TCE, necessitam de uma internação prolongada, uma vez que as vítimas precisam de um cuidado mais especial e um tempo maior de reabilitação.

Em relação aos indicadores de mortalidade, que fora dividido em óbito com menos de 24 horas e com mais de 24 horas, a taxa de mortalidade hospitalar foi de 12,9\%, isto é, 21 casos; enquanto que a taxa de mortalidade institucional foi de 10,3\%, correspondente a 4 casos, podendo-se inferir que houve uma taxa pequena de mortalidade, 
pois dos 132 prontuários avaliados somente

25 constavam óbito.

Quadro 1 - Taxa de mortalidade dos pacientes admitidos na UTIPED do HMUE no ano de 2010 ( $\mathrm{n}=132$ ).

\begin{tabular}{|c|}
\hline Indicadores de Mortalidade \\
\hline Taxa de Mortalidade Hospitalar $=12.9 \%$ - óbitos que ocorrem com mais de 24 horas \\
\hline Taxa de Mortalidade Insitucional $=\mathbf{1 0 . 3 \%}$ - óbitos que ocorrem em menos de 24 horas \\
\hline
\end{tabular}

Fonte: Prontuários da UTIPED do HMUE, 2010.

Quanto à razão para que os pacientes admitidos na UTIPED do HMUE utilizassem ventilação mecânica, teve-se como causa prevalente no estudo o rebaixamento do nível de consciência (RNC) em 73 prontuários $(86,95 \%)$, seguido pela insuficiência respiratória aguda (IRpA), em 10 prontuários
$(11,9 \%)$, e pela parada cardiorrespiratória (PCR) citada em 1 prontuário $(1,2 \%)$ (Tabela 5). Tais dados não foram similares ao estudo de Silva (2009), onde as crianças internadas em UTIPED fizeram uso de VM principalmente por IRpA, e com menos frequência por RNC.

Tabela 5 - Motivo dospacientes admitidos na UTIPED do HMUE no ano de 2010 usarem Ventilação Mecânica $(\mathrm{n}=132)$.

\begin{tabular}{lcc}
\hline \multicolumn{1}{c}{ Motivo } & \multicolumn{2}{c}{ Freqüência } \\
\cline { 2 - 3 } & $\mathbf{N}$ & $\mathbf{\%}$ \\
\hline Insuficiência respiratória & 10 & 11,9 \\
PCR & 1 & 1,2 \\
Rebaixamento de consciência & 73 & 86,9 \\
\hline Total & $\mathbf{8 4}$ & $\mathbf{1 0 0 , 0}$ \\
\hline
\end{tabular}

Fonte: Prontuários da UTIPED do HMUE, 2010.

Tal achado pode ser justificado, devido o TCE ser o principal trauma diagnosticado no ano de 2010, o qual, por sua vez desencadeia uma injúria cerebral que pode acarretar alteração do nível de consciência (sonolência,letargia, confusão mental ou coma), necessitando de admissão em unidades de tratamento intensivo e de suporte ventilatório devido a lesão causada ao sistema nervoso central.

\section{Conclusão}

O estudo contribuiu para o melhor delineamento do perfil das crianças e adolescentes vítimas de trauma na nossa região, contribuindo desta forma para que 
novos estudos sejam realizados alegando a importância de se conhecer o perfil clínico e epidemiológico das crianças e adolescentes que sofrem as graves seqüelas impostas pelo trauma.

Os dados encontrados nos ajudam também a pensar em estratégias que possam fomentar soluções para esta problemática;

\section{Referências}

1. Abramovici, S., Waksman, R. Abordagem á Criança Vítima de trauma. São Paulo: Departamento de Segurança da Criança e do Adolescente; 2005.

2. BEM, MAM. et al. Epidemiologia dos pequenos traumas em crianças atendidas no Hospital Infantil Joana de Gusmão. ArqCatarin Med. 2008 [18 abr 2011]; 59$66 . \quad$ Disponível em: $<$ http://www.acm.org.br/revista/pdf/artigos /550.pdf $>$.

3. CARVALHO, LFA. et al. Traumatismo Cranioencefalico Grave em Crianças e Adolescentes. Revista Brasileira de Terapia Intensiva,Jan-Mar 2007 [acesso em 10 jan 2011].19 (1): 98-106. Disponível em: <http://www.scielo.br/pdf/rbti/v19n1/a13v 19n1.pdf $>$.

4. Einloft PR, Garcia PC, Piva JP, Bruno F, Kipper DJ, Fiori RM. Perfil epidemiológico de dezesseis anos de uma unidade de terapia intensiva pediátrica. Rev. Saúde Pública. 2002 Dez ; 36(6): 728-733;

5. Filho VCB, Reschke CR, Horner R. Perfil epidemiológico das infecções hospitalares na Unidade de terapia intensiva infantil do Hospital de Caridade e Beneficência de Cachoeira do Sul,RS, Brasil. Revista além de reforçar a importância da atuação fisioterapêutica no tratamento destes pacientes, haja vista que a fisioterapia contribui para uma melhor reabilitação destes indivíduos, e ainda necessita de estudos que reforcem a sua importância no meio científico, em especial, dentro das Unidades de Terapia Intensiva Pediátrica.

Brasileira de Análises Clínicas. 2006; 38(4): 267-270

6. FREITAS, JPP; RIBEIRO LA; JORGE MT. Vitimas de acidentes da transito na faixa etária pediátrica atendidas em um hospital universitário: aspectos epidemiológicos e clínicos. Cad. Saúde Publica, Dez. 2007 [acesso em 03 set 2010]. 23 (12): 3055-2060. Disponível em:

$<$ http://www.scielo.br/pdf/csp/v23n12/27.p df $>$

7. LACERDA, CM. et al. Traumatismo cranioencefalico em Feira de Santana, Bahia: aspecto epidemiologicos em crianças e adolescentes. Universidade Estadual de Feira de Santana, Bahia, 2010 [acesso em 06 mar 2011] . Disponível em: $<$ http://www.uefs.br/semic/cd/resumos/297 .pdf $>$.

8. LARA, S. Assistência fisioterapeutica a pacientes politraumatizados, internados na Unidade de Terapia Intensiva (UTI) no Hospital Nossa Senhora da Conceição em Tubarão- SC, no ano de 2004, decorrente de acidente de trânsito. [Trabalho de Conclusão de Curso de Graduação em Fisioterapia]. Tubarão: Universidade do Sul de Santa Catarina, 2005. Disponível em <http://www.fisiotb.unisul.br/Tccs/SimoneLara/tcc.pdf >

9. Molina RCM et al. Caracterização das internações em uma unidade de terapia intensiva pediátrica de um hospital-escola 
da região sul do Brasil. Ciência, Cuidado e Saúde. 2008; 23(3):69-76

10. NICOLAU, MC.; LAHÓZ, AL.Fisioterapia respiratória em terapia intensiva pediátrica e neonatal: uma revisão baseada em evidências. São Paulo:Serviço de Fisioterapia do Instituto da Criança (ICR-HC-FMUSP); 2007 [acesso em 04 jun 2011].Disponível em: <http://www.pediatriasaopaulo.usp.br/uplo $\mathrm{ad} / \mathrm{pdf} / 1227 . \mathrm{pdf}>$.

11. PEREIRA, AS. et al. Determinação de fatores de risco para a queda infantil a partir do modelo calgary de avaliação familiar. RBPS, Abril./Junho, 2010 [acesso em 07 mar 2011]. Disponível em: <http://www.unifor.br/images/pdfs/rbps/art igo1_2010.2.pdf>

12. ROCHA, CG.Hospitalização por causas externas em uma unidade de terapia intensiva [Dissertação de Pós Graduação em Ciências da Saúde]. Uberlândia: Faculdade de Medicina da Universidade Federal de Uberlândia; 2007.

13. SILVA, DCB. Avaliação da ventilação mecânica utilizada em unidade de terapia intensiva pediatrica e os fatores de risco: em busca de uma melhor prática ventilatória [Dissertação de Mestrado]. São Paulo: Universidade de São Paulo, 2009 [acesso em 15 maio 2011]. Disponível em: http://www.teses.usp.br.com.br >

14.VIANA, FP. et al. Aspectos epidemiológicos das crianças com queimaduras internadas no Pronto Socorro para queimaduras de Goiânia - Goiás.Rev. Eletr. Enf. [Internet], 2009 [acesso em 14 maio 2011]; 11 (4): 779-784. Disponível em:

<http://www.fen.ufg.br/revista/v11/n4/pdf/ v11n4a02.pdf $>$. 